\title{
Circulating Level of Monocyte Chemoattractant Protein-I and Risk of Coronary Artery Disease: A Case-Control and Mendelian Randomization Study
}

\author{
Jing $\mathrm{Li}^{1}$ \\ Yanqun Zhang' \\ Xue Guo' \\ Yuanyuan $\mathrm{Wu}^{\prime}$ \\ Ruo Huang \\ Xia $\mathrm{Han}^{2}$ \\ 'Department of Health Care, Jinan \\ People's Hospital Affiliated to Shandong \\ First Medical University, Jinan, Shandong \\ Province, 27II00, People's Republic of \\ China; ${ }^{2}$ Department of Cardiology, Jinan \\ People's Hospital Affiliated to Shandong \\ First Medical University, Jinan, Shandong \\ Province, 27II00, People's Republic of \\ China
}

Correspondence: Xia Han

Department of Cardiology, Jinan People's

Hospital Affiliated to Shandong First

Medical University, Jinan, Shandong

Province, 27I I00, People's Republic of

China

Email han0890@2Icn.com
Background: Coronary artery disease (CAD) ranks the leading cause of death worldwide, and inflammation has been implicated in all stages of CAD and is considered to contribute to the pathophysiological basis of atherogenesis.

Methods: Here, we implemented a case-control study and a two-sample Mendelian randomization (MR) study to explore the associations between CAD risk and genetic predisposition to circulating level of monocyte chemoattractant protein-1 (MCP1), the most important regulator of monocyte trafficking.

Results: In case-control study, we found circulating level of MCP1 was significantly associated with increased risk of CAD (OR for per quartile increment: $1.33,95 \% \mathrm{CI}$ : $1.19-1.49, \mathrm{P}<0.001)$. Further, genetically predicted higher level of MCP1 was significantly associated with higher risk of CAD (OR for 1-SD increase: 1.05, 95\% CIs: 1.02-1.08, $P$ value: 0.002$)$ in MR analysis. Sensitivity analyses were also conducted to validate the main findings, and we also did not detect any directional pleiotropy effects using the MR Egger intercept test $(\mathrm{P}=0.831)$.

Conclusion: To sum up, our study suggested that increased CAD risk was associated with a predisposition to higher level of MCP1. Additional insight into the contribution of MCP1 to the occurrence of CAD is still needed.

Keywords: MCP1, Mendelian randomization, coronary artery disease, case-control

\section{Introduction}

Coronary artery disease (CAD), which causes about one-third of all deaths in people older than 35 years, ranks the leading cause of death worldwide., According to report of Global Burden of Diseases (GBD) 2017, 8930.4 (8790.7 to 9138.7) thousands all-age deaths occurred annually, and estimated years of life lost (YLLs) from CAD ranked first, increased by 17.3\% (15.4-19.0) from 2007 to 2017. ${ }^{3}$ Apart from conventional risk factors, like smoking, diabetes, serum total cholesterol, and systolic blood pressure, inflammation has been implicated in all stages of $\mathrm{CAD}$ and is considered to contribute to the pathophysiological basis of atherogenesis. ${ }^{4,5}$ Consequently, controlling the inflammatory response of inflammatory cytokines would be of interest as targets for primary CAD prevention. ${ }^{6}$

Previously, monocyte chemoattractant protein-1 (MCP1/CCL2) was revealed to be associated with higher risk of stroke, which indicated the possibility of targeting MCP1 or its receptors to lower the stroke incidence, using a large two-sample Mendelian randomization (MR) study. ${ }^{7} \mathrm{MCP} 1$, which is the most important 
regulator of monocyte trafficking and functions as a potent activator of mononuclear phagocytes, has been shown to be associated with peripheral arterial disease, ischemic stroke, lupus nephritis, body weight, and breast cancer. $^{6,8-12}$ A recent meta-analysis revealed that higher circulating MCP1 levels were associated with higher longterm cardiovascular mortality. ${ }^{13}$ Several studies also reported the association between $\mathrm{MCP} 1$ and $\mathrm{CAD}$ risk; however, few were conducted in Chinese and the results were inconsistent. ${ }^{14-18}$ Given this background, the causality between circulating level of MCP1 and CAD risk is still unclear and to be addressed.

Here, we first conducted a case-control study in Chinese population to explore the association of circulating level of MCP1 with CAD risk. Then, by leveraging data from the largest genome-wide association study (GWAS) of circulating level of MCP1 in in 8,293 healthy subjects of Finnish ancestry, ${ }^{19}$ and the CARDIoGRAMplusC4D consortium of $\mathrm{CAD},{ }^{20}$ we conducted a two-sample MR analysis, which aims to overcome the limitations of conventional studies (confounding bias and reverse causation), to validate the associations between circulating level of MCP1 and CAD risk.

\section{Patients and Methods Case-Control Study}

Totally 498 CAD patients and 499 healthy controls (frequency-matched by age, gender, and living areas) were included in this case-control study. Coronary angiography was performed and assessed by at least two cardiologists for CAD diagnosis with a diameter stenosis of more than $50 \%$ in any of the main coronary arteries, or a history of prior angioplasty, coronary artery bypass surgery or an MI history validated by electrocardiographic changes. Fasting venous blood is collected into a serum tube with $0.1 \%$ EDTA and stored at minus $80^{\circ}$ Celsius before analysis. Serum MCP-1 levels were determined by a multiplex assay using the Bioplex Suspension Array (Bio-Rad, Veenendaal, The Netherlands) according to the manufacturer's specifications. All samples are thawed only once and measured in triple. The study has been approved by the institutional review board of Jinan People's Hospital Affiliated to Shandong First Medical University. Informed consent was received from all the study participants, and the guidelines outlined in the Declaration of Helsinki were followed.
Statistical analyses were carried out using IBM SPSS Statistics version 22.0, while two-tailed P-values $<0.05$ were considered significant. Data are presented as mean $\pm \mathrm{SD}$ for continuous variables and as proportions for categorical variables. Quartiles of MCP-1 distribution among controls were used to convert it to a categorical variable, and the associations between MCP-1 quartiles and $\mathrm{CAD}$ risk were assessed using logistic regression analyses.

\section{MR Analyses}

The genetic instruments for circulating level of MCP1 was selected using the following criteria: 1) a genome-wide threshold of significance of $\mathrm{p}<5 \times 10^{-8}, 2$ ) for all SNPs in linkage disequilibrium (LD; $r^{2}<0.6$ in the European $1000 \mathrm{G}$ reference panel), we also retain tagSNPs as independents instrument. Finally, 36 SNPs were adopted as final instruments (Supplementary Table 1). Summary statistics for the associations of the identified instruments with CAD risk were extracted from the CARDIoGRAMplusC4D, which assembled 60,801 cases and 123,504 control subjects for 48 studies. $^{20}$ MR was performed using $\mathrm{R}$ version 4.0 statistical software, package "TwoSampleMR", (The $\mathrm{R}$ Foundation for Statistical Computing, Vienna, Austria). The primary MR analysis was conducted by using inverse-variance weighted (IVW) regression analysis. We used the intercept obtained from the MR-Egger regression as a measure of directional pleiotropy.

\section{Results}

\section{Case-Control Study}

Table 1 presents the clinical characteristics of CAD cases and controls. The results showed that there is no significant difference for the distribution of age, gender, drinking status, diabetes, and hypertension $(\mathrm{P}>0.05)$. However, the cases are more likely to be smokers, compared with the controls $(\mathrm{P}<0.001)$. The cases had significantly higher circulating level of MCP1 (Mean \pm SD: $69.7 \pm 21.2$ vs $61.2 \pm 18.7 ; \mathrm{P}<0.001)$. When analyzed as a continuous variable, circulating level of MCP1 was significantly associated with increased risk of CAD (OR for per unit: 1.02, 95\% CI: 1.01-1.03, $\mathrm{P}<0.001$, Table 2). When analyzed as a categorical variable, circulating level of MCP1 was significantly associated with increased risk of CAD (OR for per quartile increment: 1.33 , 95\% CI: $1.19-1.49$, $\mathrm{P}<0.001$, Table 2). Compared with the first quartile, all of the second (OR: 1.47; 95\% CIs: 1.01-2.16), the third (OR: 1.79; 95\% 
Table I Clinical Demographic Characteristics of CAD Cases and Controls

\begin{tabular}{|c|c|c|c|}
\hline Variables & $\begin{array}{c}\text { Cases } \\
(n=498)\end{array}$ & $\begin{array}{l}\text { Controls } \\
(n=499)\end{array}$ & $P$ value \\
\hline \multicolumn{4}{|l|}{ Age (years) } \\
\hline$\geq 60$ & $231(46.4 \%)$ & $242(48.5 \%)$ & 0.692 \\
\hline$<60$ & $258(53.6 \%)$ & 257 (5I.5\%) & \\
\hline \multicolumn{4}{|l|}{ Gender } \\
\hline Male & 347 (69.7\%) & 345 (69.1\%) & 0.853 \\
\hline Female & $15 \mathrm{I}(30.3 \%)$ & 154 (30.9\%) & \\
\hline \multicolumn{4}{|l|}{ Smoking status } \\
\hline Smokers & $211(42.4 \%)$ & $133(26.7 \%)$ & $<0.001$ \\
\hline Non-smokers & $287(57.6 \%)$ & 366 (73.3\%) & \\
\hline \multicolumn{4}{|l|}{ Drinking status } \\
\hline Drinkers & $123(24.7 \%)$ & $101(20.2 \%)$ & 0.092 \\
\hline Non-drinkers & 375 (75.3\%) & 398 (79.8\%) & \\
\hline \multicolumn{4}{|l|}{ Diabetes } \\
\hline Yes & $100(20.0 \%)$ & 79 (I5.8\%) & $0.08 I$ \\
\hline No & $398(80.0 \%)$ & $420(84.2 \%)$ & \\
\hline \multicolumn{4}{|l|}{ Hypertension } \\
\hline Yes & $276(55.4 \%)$ & $25 \mathrm{I}(50.3 \%)$ & 0.105 \\
\hline No & $222(44.6 \%)$ & $248(49.7 \%)$ & \\
\hline MCPI (pg/mL) & $69.7 \pm 21.2$ & $61.2 \pm 18.7$ & $<0.001$ \\
\hline
\end{tabular}

Table 2 Distribution of Quartiles of MCPI in CAD Cases and Controls

\begin{tabular}{|c|c|c|c|c|}
\hline IF $-\gamma(\mathrm{pg} / \mathrm{mL})$ & Cases & Controls & OR (95\% Cls)* & $P$ value \\
\hline Quartile I ( $\leq 45.1)$ & 78 & 125 & Reference & \\
\hline $\begin{array}{l}\text { Quartile } 2 \\
(45.1-61.6)\end{array}$ & 115 & 125 & $1.47(1.01-2.16)$ & 0.045 \\
\hline $\begin{array}{l}\text { Quartile } 3 \\
(61.6-76.5)\end{array}$ & 107 & 125 & $1.79(0.94-2.01)$ & 0.106 \\
\hline Quartile 4 (>76.5) & 198 & 124 & $3.98(1.78-3.67)$ & $<0.001$ \\
\hline $\begin{array}{l}\text { Per unit (continuous } \\
\text { variable) }\end{array}$ & & & $1.02(1.01-1.03)$ & $<0.001$ \\
\hline Per quartile & & & $1.33(1.19-1.49)$ & $<0.001$ \\
\hline
\end{tabular}

Note: *Adjusted for age, gender, and smoking status.

CIs: 0.94-2.01), and the fourth (OR: 3.98; 95\% CIs: 1.78-3.67) quartiles were associated with increased risk of CAD.

\section{MR Analyses}

In the MR analysis, 36 SNPs were adopted as final instruments (Supplementary Table 1 shows the associations between each SNP and MCP-1, as well as risk of CAD), and the $F$-statistics ranged from 30.3 to 157.5 , suggesting the non-existence of weak instruments bias. Totally, these individual SNPs could explain $21.6 \%$ of the variance of MCP-1 levels. As shown in Figure 1, genetically predicted higher level of MCP1 was significantly associated with higher risk of CAD (OR for 1-SD increase: 1.05 , 95\% CIs: $1.02-1.08$, P value: 0.002 ), when analyzed using the IVW model. Sensitivity analyses were also conducted using the weighted median, maximum likelihood, penalized weighted median, IVW radial, and the sign concordance test; the results were not materially changed (data not shown). There was no evidence for heterogeneity by Cochran $\mathrm{Q}$ test $(\mathrm{P}=0.234)$ and no outlier SNPs were detected with the MR-PRESSO test. Further, we also did not detect any directional pleiotropy effects using the MR Egger intercept test $(\mathrm{P}=0.831)$. The leave-one-out plot provided additional support that no single SNP drove the overall association with CAD risk (Figure 2).

\section{Discussion}

In the current study, a case-control study and a twosample MR approach were conducted to comprehensively evaluate the causal relationships between genetic predisposition to circulating level of MCP1 and CAD risk. Using case-control design, we consistently found circulating level of MCP1 was significantly associated with increased risk of CAD. Further, genetically predicted higher level of MCP1 (1-SD increase) was significantly associated with increased risk of CAD using two-sample MR approach. To sum up, our results highlighted that circulating level of MCP1 contributed to the risk of CAD.

Due to inflammation was the underlying pathophysiological causal of atherosclerosis, chemokines, produced in the atherosclerotic vessel, has been explored as prime biomarkers of CAD for a long period. ${ }^{21} \mathrm{MCP} 1$, involved in the formation, progression, destabilization of atheromatous plaques and postinfarction remodeling, was first reported by Yoshimura et al in $1989,{ }^{22}$ and identified to mediate monocytic infiltration of the artery wall. ${ }^{23}$ Except for stroke and CAD, it has been also linked to idiopathic pulmonary fibrosis, rheumatoid arthritis, pulmonary granulomatosis, lupus nephritis, diabetes, cancers, atrial fibrillation, neointimal hyperplasia, etc. ${ }^{24-30}$ The $-2518 \mathrm{~A} / \mathrm{G}$ polymorphism in MCP-1 gene was also associated with diabetes risk. ${ }^{31} \mathrm{MCP} 1$ is involved in the genesis, progression, inflammatory response and immune process of CAD, which suggests the potential as a liquid biomarker of $\mathrm{CAD}^{32}$ Although meta-analysis revealed that higher circulating MCP-1 levels were associated with higher long- 


\section{MR Test}
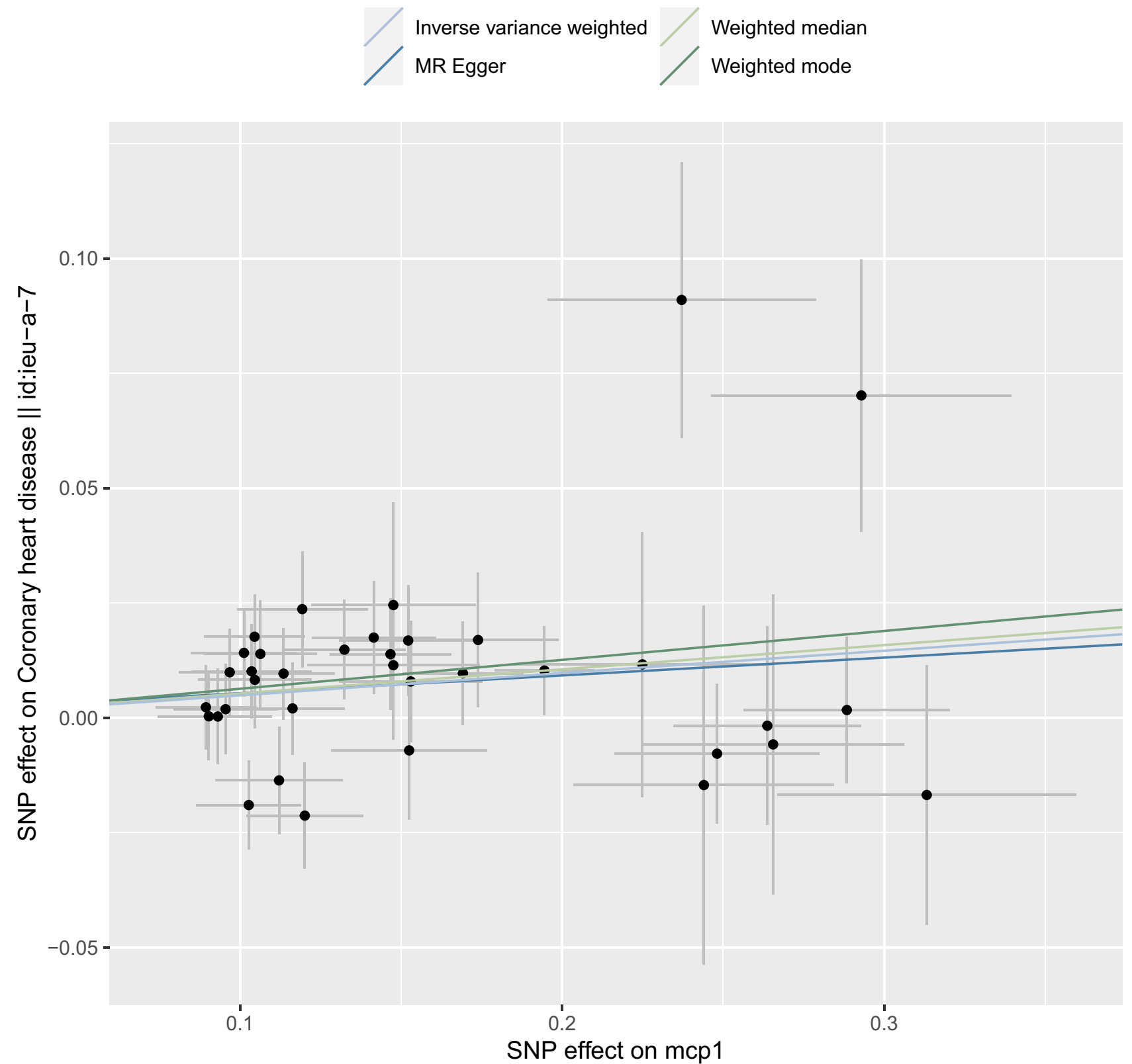

Figure I Scatter plot of SNP potential effects of genetic predisposition to circulating level of MCPI on CAD risk, with the slope of each line corresponding to estimated MR effect per method.

term cardiovascular mortality and increased long-term risk of stroke, its association with CAD risk was still inconsistent and few were conducted in Chinese. ${ }^{11,13}$

The use of MR, which is less affected by issues of confounding, reverse causation and measurement error bias, is particularly useful in this context, as genetic variations closely related to the circulating level of MCP1 can be used as instrumental variables to assess their causal role in the development of CAD. ${ }^{10}$ Previously, Georgakis et $\mathrm{al}^{7}$ found circulating levels of MCP-1 were associated with higher risk of stroke, in particular with large-artery stroke and cardioembolic stroke. Li et al ${ }^{10}$ reported that genetically predicted circulating levels (1-SD increase) of MCP1 were significantly associated with increased risk of overall breast cancer, as well as ER-positive breast cancer. In our study, we found genetically predicted higher level of MCP1 was significantly associated with higher risk of CAD (OR for 1-SD increase: 1.05, 95\% CIs: 1.02-1.08, 


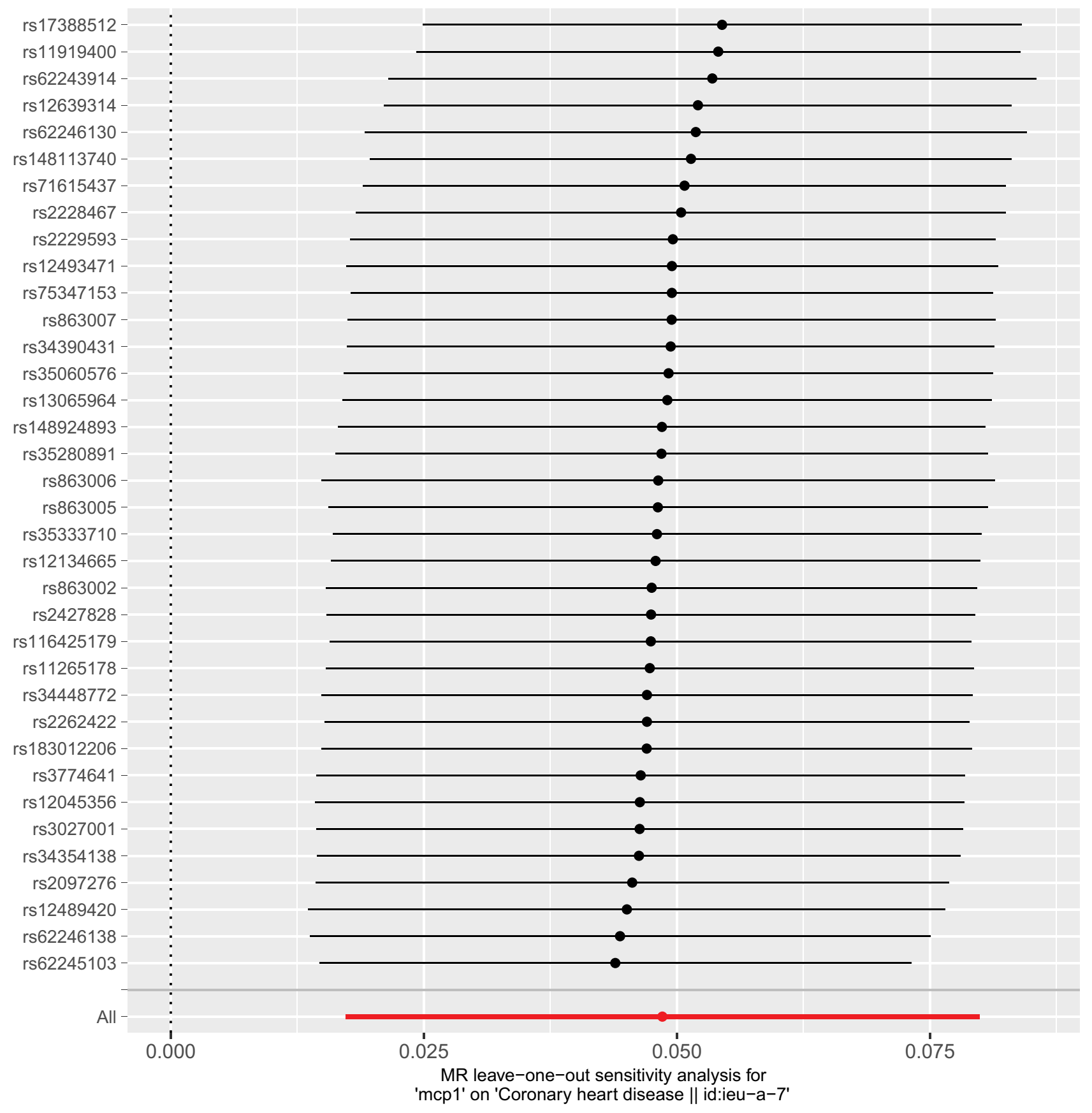

Figure 2 The leave-one-out plot for association between genetic predisposition to circulating level of MCPI and CAD risk.

P value: 0.002$)$, using the IVW model and other sensitivity analysis models. These findings enhanced the strength of argument for observational studies.

The strength of the current study includes the cross validation of the case-control study and MR analysis, and the enough statistical power (for case-control study: 100\%; for MR analysis: $99.6 \%$ ). However, one limitation should be also considered when interpreting these results. The case-control study and MR analysis were conducted in different populations, though the association of circulating level of MCP1 with CAD risk should not be changed by ethnicity issue, just like other serum biomarkers (CPR, lipids, etc). This is because our sample size was not statistically efficient enough to conduct an MR analysis.

Conclusively, our study provided strong evidence for MCP1 as a causal risk factor for CAD. Both case-control study and MR analysis suggested that increased CAD risk was associated with a pre-disposition to higher level of 
MCP1. To the best of our knowledge, this should be the first study to examine the causal effect of MCP1 on risk of CAD, using MR. Additional insight into the contribution of MCP1 to the occurrence of CAD is still needed.

\section{Disclosure}

The authors declare that they have no conflicts of interest.

\section{References}

1. Siegel RL, Miller KD, Jemal A. Cancer statistics, 2020. CA Cancer J Clin. 2020;70(1):7-30. doi:10.3322/caac.21590

2. Sanchis-Gomar F, Perez-Quilis C, Leischik R, Lucia A. Epidemiology of coronary heart disease and acute coronary syndrome. Ann Transl Med. 2016;4(13):256. doi:10.21037/ atm.2016.06.33

3. Collaborators GBDCoD. Global, regional, and national age-sexspecific mortality for 282 causes of death in 195 countries and territories, 1980-2017: a systematic analysis for the global burden of disease study 2017. Lancet. 2018;392(10159):1736-1788.

4. Okami Y, Ueshima H, Nakamura Y, et al.; Group NDR. Risk factors that most accurately predict coronary artery disease based on the duration of follow-up- NIPPON DATA80. Circ J. 2020. doi:10.1253/circj.CJ-20-0739

5. Harrington RA. Targeting inflammation in coronary artery disease. N Engl J Med. 2017;377(12):1197-1198. doi:10.1056/NEJMe1709904

6. Lim SB, Di Lee W, Vasudevan J, Lim WT, Lim CT. Liquid biopsy: one cell at a time. NPJ Precis Oncol. 2019;3:23. doi:10.1038/s41698019-0095-0

7. Georgakis MK, Gill D, Rannikmae K, et al. Genetically determined levels of circulating cytokines and risk of stroke. Circulation. 2019;139(2):256-268. CIRCULATIONAHA.118.035905

8. Abozaid MA, Ahmed GH, Tawfik NM, Sayed SK, Ghandour AM, Madkour RA. Serum and urine monocyte chemoattractant protein-1 as a markers for lupus nephritis. Egypt J Immunol. 2020;27(1):97-107.

9. Huang HH, Chen LY, Chen KY, Lee YC, Tsai CY, Chen CY. Increased monocyte chemoattractant protein- 1 and nitrotyrosine are associated with increased body weight in patients with rheumatoid arthritis after etanercept therapy. Neuropeptides. 2020;84:102100. doi:10.1016/j.npep.2020.102100

10. Li S, Xu Y, Zhang Y, et al. Mendelian randomization analyses of genetically predicted circulating levels of cytokines with risk of breast cancer. NPJ Precis Oncol. 2020;4:25. doi:10.1038/s41698020-00131-6

11. Georgakis MK, Malik R, Bjorkbacka H, et al. Circulating monocyte chemoattractant protein-1 and risk of stroke: meta-analysis of population-based studies involving 17180 individuals. Circ Res. 2019;125(8):773-782. doi:10.1161/CIRCRESAHA.119.315380

12. Bin $\lim$ S, Chua MLK, Yeong JPS, Tan SJ, Lim WT, Lim CT. Pancancer analysis connects tumor matrisome to immune response. NPJ Precis Oncol. 2019;3:15. doi:10.1038/s41698-019-0087-0

13. Georgakis MK, de Lemos JA, Ayers C, et al. Association of circulating monocyte chemoattractant protein-1 levels with cardiovascular mortality: a meta-analysis of population-based studies. JAMA Cardiol. 2020. doi:10.1001/jamacardio.2020.5392

14. Hoogeveen RC, Morrison A, Boerwinkle E, et al. Plasma MCP-1 level and risk for peripheral arterial disease and incident coronary heart disease: atherosclerosis risk in communities study. Atherosclerosis. 2005;183(2):301-307. doi:10.1016/j.atherosclerosis. 2005.03.007
15. Kidd LR, Jones DZ, Rogers EN, et al. Chemokine Ligand 5 (CCL5) and chemokine receptor (CCR5) genetic variants and prostate cancer risk among men of African Descent: a case-control study. Hered Cancer Clin Pract. 2012;10(1):16. doi:10.1186/1897-4287-10-16

16. van Wijk DF, van Leuven SI, Sandhu MS, et al. Chemokine ligand 2 genetic variants, serum monocyte chemoattractant protein-1 levels, and the risk of coronary artery disease. Arterioscler Thromb Vasc Biol. 2010;30(7):1460-1466. doi:10.1161/ATVBAHA.110.205526

17. Canoui-Poitrine F, Luc G, Mallat Z, et al. Systemic chemokine levels, coronary heart disease, and ischemic stroke events: the PRIME study. Neurology. 2011;77(12):1165-1173. doi:10.1212/WNL.0b013e318 $22 \mathrm{dc} 7 \mathrm{c} 8$

18. Schiopu A, Bengtsson E, Goncalves I, Nilsson J, Fredrikson GN, Bjorkbacka H. Associations between macrophage colony-stimulating factor and monocyte chemotactic protein 1 in plasma and first-time coronary events: a nested case-control study. J Am Heart Assoc. 2016;5(9). doi:10.1161/JAHA.115.002851

19. Ahola-Olli AV, Wurtz P, Havulinna AS, et al. Genome-wide association study identifies 27 Loci influencing concentrations of circulating cytokines and growth factors. Am J Hum Genet. 2017;100(1):40-50. doi:10.1016/j.ajhg.2016.11.007

20. Deloukas P, Kanoni S, Willenborg C, et al. Large-scale association analysis identifies new risk loci for coronary artery disease. Nat Genet. 2013;45(1):25-33. doi:10.1038/ng.2480

21. Ardigo D, Assimes TL, Fortmann SP, et al. Circulating chemokines accurately identify individuals with clinically significant atherosclerotic heart disease. Physiol Genomics. 2007;31(3):402-409. doi:10.1152/physiolgenomics.00104.2007

22. Yoshimura T, Yuhki N, Moore SK, Appella E, Lerman MI, Leonard EJ. Human monocyte chemoattractant protein-1 (MCP-1). Full-length cDNA cloning, expression in mitogen-stimulated blood mononuclear leukocytes, and sequence similarity to mouse competence gene JE. FEBS Lett. 1989;244(2):487-493. doi:10.1016/00145793(89)80590-3

23. Nelken NA, Coughlin SR, Gordon D, Wilcox JN. Monocyte chemoattractant protein-1 in human atheromatous plaques. J Clin Invest. 1991;88(4):1121-1127. doi:10.1172/JCI115411

24. Antoniades HN, Neville-Golden J, Galanopoulos T, Kradin RL, Valente AJ, Graves DT. Expression of monocyte chemoattractant protein $1 \mathrm{mRNA}$ in human idiopathic pulmonary fibrosis. Proc Natl Acad Sci U S A. 1992;89(12):5371-5375. doi:10.1073/ pnas.89.12.5371

25. Koch AE, Kunkel SL, Harlow LA, et al. Enhanced production of monocyte chemoattractant protein-1 in rheumatoid arthritis. J Clin Invest. 1992;90(3):772-779. doi:10.1172/JCI115950

26. Bonner ER, Bornhorst M, Packer RJ, Nazarian J. Liquid biopsy for pediatric central nervous system tumors. NPJ Precis Oncol. 2018;2:29. doi:10.1038/s41698-018-0072-z

27. Kim S, Han Y, Kim SI, Kim HS, Kim SJ, Song YS. Tumor evolution and chemoresistance in ovarian cancer. NPJ Precis Oncol. 2018;2:20. doi:10.1038/s41698-018-0063-0

28. Urrego-Callejas T, Alvarez SS, Arias LF, et al. Urinary levels of ceruloplasmin and monocyte chemoattractant protein-1 correlate with extra-capillary proliferation and chronic damage in patients with lupus nephritis. Clin Rheumatol. 2020. doi:10.1007/s10067-020-05454-0

29. Savelieva I, Camm AJ. A new biomarker in atrial fibrillation: monocyte chemoattractant protein-1-induced protein. Cardiology. 2019;144(3-4):122-124. doi:10.1159/000502253

30. Egashira K, Zhao Q, Kataoka C, et al. Importance of monocyte chemoattractant protein-1 pathway in neointimal hyperplasia after periarterial injury in mice and monkeys. Circ Res. 2002;90 (11):1167-1172. doi:10.1161/01.RES.0000020561.03244.7E 
31. Zhang Y, Zhang J, Tian C, et al. The $-2518 \mathrm{~A} / \mathrm{G}$ polymorphism in the monocyte chemoattractant protein-1 (MCP-1) gene and diabetes risk: a meta-analysis. Diabetes Res Clin Pract. 2011;94(3):e89-92. doi:10. 1016/j.diabres.2011.09.005
32. Li M, Chen Y, Zhang Y, Li D, Liu J. Correlation between monocyte chemoattractant protein-1/chemokine (C-C motif) ligand 2 and coronary plaque characteristics. Exp Biol Med. 2020;245(15):13 35-1343. doi:10.1177/1535370220941424

\section{Publish your work in this journal}

Pharmacogenomics and Personalized Medicine is an international, peer-reviewed, open access journal characterizing the influence of genotype on pharmacology leading to the development of personalized treatment programs and individualized drug selection for improved safety, efficacy and sustainability. This journal is indexed on the American Chemical Society's Chemical Abstracts Service (CAS). The manuscript management system is completely online and includes a very quick and fair peer-review system, which is all easy to use. Visit http://www.dovepress.com/testimonials.php to read real quotes from published authors. 